University of Texas at El Paso

ScholarWorks@UTEP

$5-2003$

\title{
Outlier Detection under Interval and Fuzzy Uncertainty: Algorithmic Solvability and Computational Complexity
}

\author{
Vladik Kreinovich \\ The University of Texas at El Paso, vladik@utep.edu \\ Praveen Patangay \\ Luc Longpre \\ The University of Texas at El Paso, longpre@utep.edu \\ Scott A. Starks \\ The University of Texas at El Paso, sstarks@utep.edu \\ Cynthia Campos
}

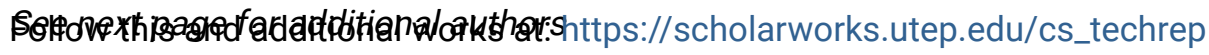 \\ Part of the Computer Engineering Commons}

Comments:

Technical Report: UTEP-CS-03-10d

Published in: Proceedings of the Annual Conference of the North American Fuzzy Information

Processing Society NAFIPS'03, Chicago, Illinois, July 24-26, 2003, pp. 410-406.

\section{Recommended Citation}

Kreinovich, Vladik; Patangay, Praveen; Longpre, Luc; Starks, Scott A.; Campos, Cynthia; Ferson, Scott; and Ginzburg, Lev, "Outlier Detection under Interval and Fuzzy Uncertainty: Algorithmic Solvability and Computational Complexity" (2003). Departmental Technical Reports (CS). 285.

https://scholarworks.utep.edu/cs_techrep/285

This Article is brought to you for free and open access by the Computer Science at ScholarWorks@UTEP. It has been accepted for inclusion in Departmental Technical Reports (CS) by an authorized administrator of ScholarWorks@UTEP. For more information, please contact Iweber@utep.edu. 


\section{Authors}

Vladik Kreinovich, Praveen Patangay, Luc Longpre, Scott A. Starks, Cynthia Campos, Scott Ferson, and Lev Ginzburg 


\section{Outlier Detection Under Interval and Fuzzy Uncertainty: Algorithmic Solvability and Computational Complexity}

\author{
Vladik Kreinovich, Praveen Patangay \\ Luc Longpré, Scott A. Starks, Cynthia Campos \\ NASA Pan-American Center for Earth and Environmental Studies \\ University of Texas at El Paso \\ El Paso, TX 79968, USA \\ vladik@cs.utep.edu
}

\author{
Scott Ferson, Lev Ginzburg \\ Applied Biomathematics \\ 100 North Country Road \\ Setauket, NY 11733, USA \\ scott@ramas.com
}

\begin{abstract}
In many application areas, it is important to detect outliers. Traditional engineering approach to outlier detection is that we start with some "normal" values $x_{1}, \ldots, x_{n}$, compute the sample average $E$, the sample standard variation $\sigma$, and then mark a value $x$ as an outlier if $x$ is outside the $k_{0}$-sigma interval $\left[E-k_{0} \cdot \sigma, E+k_{0} \cdot \sigma\right]$ (for some pre-selected parameter $k_{0}$ ). In real life, we often have only interval ranges $\left[\underline{x}_{i}, \bar{x}_{i}\right]$ for the normal values $x_{1}, \ldots, x_{n}$. In this case, we only have intervals of possible values for the bounds $E-k_{0} \cdot \sigma$ and $E+k_{0} \cdot \sigma$. We can therefore identify outliers as values that are outside all $k_{0}$-sigma intervals.

In this paper, we analyze the computational complexity of these outlier detection problems, and provide efficient algorithms that solve some of these problems (under reasonable conditions).

We also provide algorithms that estimate the degree of "outlier-ness" of a given value $x$-measured as the largest value $k_{0}$ for which $x$ is outside the corresponding $k_{0}$-sigma interval.
\end{abstract}

\section{Introduction}

Detecting outliers is important. In many application areas, it is important to detect outliers, i.e., unusual, abnormal values; e.g.:

- in medicine, unusual values may indicate disease (see, e.g., [7]);

- in geophysics, abnormal values may indicate a mineral deposit or an erroneous measurement result (see, e.g., $[5,9,13,16])$;

- in structural integrity testing, abnormal values may indicate faults in a structure $[2,6,7,10,11,17])$.
Traditional approach to outlier detection. Traditional engineering approach to outlier detection (see, e.g., [1, 12, $15])$ is as follows:

- first, we collect measurement results $x_{1}, \ldots, x_{n}$ corresponding to normal situations;

- then, we compute the sample average $E \stackrel{\text { def }}{=}$ $\frac{x_{1}+\ldots+x_{n}}{n}$ of these normal values and the (sample) standard deviation $\sigma=\sqrt{V}$, where $V \stackrel{\text { def }}{=}$ $\frac{\left(x_{1}-E\right)^{2}+\ldots+\left(x_{n}-E\right)^{2}}{n}$;

- finally, a new measurement result $x$ is classified as an outlier if it is outside the interval $[L, U]$ (i.e., if either $x<L$ or $x>U$ ), where $L \stackrel{\text { def }}{=} E-k_{0} \cdot \sigma, U \stackrel{\text { def }}{=}$ $E+k_{0} \cdot \sigma$, and $k_{0}>1$ is some pre-selected value (most frequently, $k_{0}=2,3$, or 6 ).

Fuzzy uncertainty. Instead of arbitrarily selecting $k_{0}$ and classifying a value as an outlier or not an outlier, it is reasonable to treat the set of outliers as a fuzzy set and to return the degree of confidence to which each value is an outlier: if the corresponding $k_{0}$ is 6 , this degree is close to 1 ; if the corresponding $k_{0}$ is 1 , this degree is close to 0 .

Interval uncertainty. In some practical situations, we only have intervals $\mathbf{x}_{i}=\left[\underline{x}_{i}, \bar{x}_{i}\right]$ of possible values of $x_{i}$. This happens, for example, if instead of observing the actual value $x_{i}$ of the random variable, we observe the value $\widetilde{x}_{i}$ measured by an instrument with a known upper bound $\Delta_{i}$ on the measurement error; then, the actual (unknown) value is within the interval $\mathbf{x}_{i}=\left[\widetilde{x}_{i}-\Delta_{i}, \widetilde{x}_{i}+\Delta_{i}\right]$. For different values $x_{i} \in \mathbf{x}_{i}$, we get different bounds $L$ and $U$. Possible values of $L$ form an interval - we will denote it by $\mathbf{L} \stackrel{\text { def }}{=}[\underline{L}, \bar{L}]$; possible values of $U$ form an interval $\mathbf{U}=[\underline{U}, \bar{U}]$. 
Possible and guaranteed outliers. How do we now detect outliers? There are two possible approaches to this question: we can detect possible outliers and we can detect guaranteed outliers:

- a value $x$ is a possible outlier if it is located outside one of the possible $k_{0}$-sigma intervals $[L, U]$ (but is may be inside some other possible interval $[L, U]$ );

- a value $x$ is a guaranteed outlier if it is located outside all possible $k_{0}$-sigma intervals $[L, U]$.

Which approach is more reasonable depends on a possible situation:

- if our main objective is not to miss an outlier, e.g., in structural integrity tests, when we do not want to risk launching a spaceship with a faulty part, it is reasonable to look for possible outliers;

- if we want to make sure that the value $x$ is an outlier, e.g., if we are planning a surgery and we want to make sure that there is a micro-calcification before we start cutting the patient, then we would rather look for guaranteed outliers.

The two approaches can be described in terms of the endpoints of the intervals $\mathbf{L}$ and $\mathbf{U}$ :

A value $x$ guaranteed to be normal - i.e., it is not a possible outlier - if $x$ belongs to the intersection of all possible intervals $[L, U]$; the intersection corresponds to the case when $L$ is the largest and $U$ is the smallest, i.e., this intersection is the interval $[\bar{L}, \underline{U}]$. So, if $x>\underline{U}$ or $x<\bar{L}$, then $x$ is a possible outlier, else it is guaranteed to be a normal value.

If a value $x$ is inside one of the possible intervals $[L, U]$, then it can still be normal; the only case when we are sure that the value $x$ is an outlier is when $x$ is outside all possible intervals $[L, U]$, i.e., is the value $x$ does not belong to the union of all possible intervals $[L, U]$ of normal values; this union is equal to the interval $[\underline{L}, \bar{U}]$. So, if $x>\bar{U}$ or $x<\underline{L}$, then $x$ is a guaranteed outlier, else it can be a normal value.

Comment. In real life, the situation may be slightly more complicated because, as we have mentioned, measurements often come with interval inaccuracy; so, instead of the exact value $x$ of the measured quantity, we get an interval $\mathbf{x}=$ $[\underline{x}, \bar{x}]$ of possible values of this quantity.

In this case, we have a slightly more complex criterion for outlier detection:

- the actual (unknown) value of the measured quantity is a possible outlier if some value $x$ from the interval $[\underline{x}, \bar{x}]$ is a possible outlier, i.e., is outside the intersection $[\bar{L}, \underline{U}]$; thus, the value is a possible outlier if one of the two inequalities hold: $\underline{x}<\bar{L}$ or $\underline{U}<\bar{x}$.
- the actual (unknown) value of the measured quantity is guaranteed to be an outlier if all possible values $x$ from the interval $[\underline{x}, \bar{x}]$ are guaranteed to be outliers (i.e., are outside the union $[\underline{L}, \bar{U}]$ ); thus, the value is a guaranteed outlier if one of the two inequalities hold: $\bar{x}<\underline{L}$ or $\bar{U}<\underline{x}$.

In all these cases:

- to detect possible outliers, we must be able to compute the values $\bar{L}$ and $\underline{U}$;

- to detect guaranteed outliers, we must be able to compute the values $\underline{L}$ and $\bar{U}$.

What we are planning to do. In this paper, we analyze the computational complexity of these outlier detection problems and provide efficient algorithms that solve these problems (under reasonable conditions).

\section{What Was Known Before}

As we discussed in the introduction, to detect outliers under interval uncertainty, we must be able to compute the range $\mathbf{L}=[\underline{L}, \bar{L}]$ of possible values of $L=E-k_{0} \cdot \sigma$ and the range $\mathbf{U}=[\underline{U}, \bar{U}]$ of possible values of $U=E+k_{0} \cdot \sigma$.

In $[3,4]$, we have shown how to compute the intervals $\mathbf{E}=[\underline{E}, \bar{E}]$ and $[\underline{\sigma}, \bar{\sigma}]$ of possible values for $E$ and $\sigma$. In principle, we can use the general ideas of interval computations to combine these intervals and conclude, e.g., that $L$ always belongs to the interval $\mathbf{E}-k_{0} \cdot[\underline{\sigma}, \bar{\sigma}]$. However, as often happens in interval computations, the resulting interval for $L$ is wider than the actual range - wider because the values $E$ and $\sigma$ are computed based on the same inputs $x_{1}, \ldots, x_{n}$ and cannot, therefore, change independently.

We mark a value $x$ as an outlier if it is outside the interval $[L, U]$. Thus, if, instead of the actual ranges for $L$ and $U$, we use wider intervals, we may miss some outliers. It is therefore important to compute the exact ranges for $L$ and $U$. In this paper, we show how to compute these exact ranges.

\section{Detecting Possible Outliers}

To find possible outliers, we must know the values $\underline{U}$ and $\bar{L}$. In this section, we design feasible algorithms for computing the exact lower bound $\underline{U}$ of the function $U$ and the exact upper bound $\bar{L}$ of the function $L$. Specifically, our algorithms are quadratic-time, i.e., require $O\left(n^{2}\right)$ computational steps (arithmetic operations or comparisons) for $n$ interval data points $\mathbf{x}_{i}=\left[\underline{x}_{i}, \bar{x}_{i}\right]$.

The algorithms $\underline{\mathcal{A}}_{U}$ for computing $\underline{U}$ and $\overline{\mathcal{A}}_{L}$ for computing $\bar{L}$ are as follows: 
- In both algorithms, first, we sort all $2 n$ values $\underline{x}_{i}, \bar{x}_{i}$ into a sequence $x_{(1)} \leq x_{(2)} \leq \ldots \leq x_{(2 n)}$; take $x_{(0)}=-\infty$ and $x_{(2 n+1)}=+\infty$. Thus, the real line is divided into $2 n+1$ zones $\left(x_{(0)}, x_{(1)}\right],\left[x_{(1)}, x_{(2)}\right], \ldots$, $\left[x_{(2 n-1)}, x_{(2 n)}\right],\left[x_{(2 n)}, x_{(2 n+1)}\right)$.

- For each of these zones $\left[x_{(k)}, x_{(k+1)}\right], \quad k=$ $0,1, \ldots, 2 n$, we compute the values

$$
\begin{gathered}
e_{k} \stackrel{\text { def }}{=} \sum_{i: \underline{x}_{i} \geq x_{(k+1)}} \underline{x}_{i}+\sum_{j: \bar{x}_{j} \leq x_{(k)}} \bar{x}_{j}, \\
m_{k} \stackrel{\text { def }}{=} \sum_{i: \underline{x}_{i} \geq x_{(k+1)}}\left(\underline{x}_{i}\right)^{2}+\sum_{j: \bar{x}_{j} \leq x_{(k)}}\left(\bar{x}_{j}\right)^{2},
\end{gathered}
$$

and $n_{k}=$ the total number of such $i$ 's and $j$ 's. Then, we solve the quadratic equation

$$
A-B \cdot \mu+C \cdot \mu^{2}=0,
$$

where

$$
\begin{gathered}
A \stackrel{\text { def }}{=} e_{k}^{2} \cdot\left(1+\alpha^{2}\right)-\alpha^{2} \cdot m_{k} \cdot n ; \alpha \stackrel{\text { def }}{=} 1 / k_{0}, \\
B \stackrel{\text { def }}{=} 2 \cdot e_{k} \cdot\left(\left(1+\alpha^{2}\right) \cdot n_{k}-\alpha^{2} \cdot n\right) ; \\
C \stackrel{\text { def }}{=} n_{k} \cdot\left(\left(1+\alpha^{2}\right) \cdot n_{k}-\alpha^{2} \cdot n\right)
\end{gathered}
$$

For computing $\underline{U}$, we select only those solutions for which $\mu \cdot n_{k} \leq e_{k}$ and $\mu \in\left[x_{(k)}, x_{(k+1)}\right]$; for computing $\bar{L}$, we select only those solutions for which $\mu \cdot n_{k} \geq e_{k}$ and $\mu \in\left[x_{(k)}, x_{(k+1)}\right]$. For each selected solution, we compute the values of

$$
E_{k}=\frac{e_{k}}{n}+\frac{n-n_{k}}{n} \cdot \mu, \quad M_{k}=\frac{m_{k}}{n}+\frac{n-n_{k}}{n} \cdot \mu^{2},
$$

and, correspondingly,

$$
U_{k}=E_{k}+k_{0} \cdot \sqrt{M_{k}-\left(E_{k}\right)^{2}}
$$

or

$$
L_{k}=E_{k}-k_{0} \cdot \sqrt{M_{k}-\left(E_{k}\right)^{2}}
$$

- Finally, if we are computing $\underline{U}$, we return the smallest of the values $U_{k}$;

if we are computing $\bar{L}$, we return the smallest of the values $L_{k}$.

Theorem 1 The algorithms $\underline{\mathcal{A}}_{U}$ and $\overline{\mathcal{A}}_{L}$ always compute $\underline{U}$ and $\bar{L}$ in quadratic time.

Comment. The main idea of this proof is given in the last (Proofs) section. The detailed proofs are given in http://www.cs.utep.edu/vladik/2003/tr03-10c.ps.gz and in http://www.cs.utep.edu/vladik/2003/tr03-10c.pdf

\section{In General, Detecting Guaranteed Outliers is NP-Hard}

As we have mentioned in Section 1, to be able to detect guaranteed outliers, we must be able to compute the values $\underline{L}$ and $\bar{U}$. In general, this is an NP-hard problem:

Theorem 2 For every $k_{0}>1$, computing the upper endpoint $\bar{U}$ of the interval $[\underline{U}, \bar{U}]$ of possible values of $U=$ $E+k_{0} \cdot \sigma$ is NP-hard.

Theorem 3 For every $k_{0}>1$, computing the lower endpoint $\underline{L}$ of the interval $[\underline{L}, \bar{L}]$ of possible values of $L=$ $E-k_{0} \cdot \sigma$ is NP-hard.

Comment. For interval data, the NP-hardness of computing the upper bound for $\sigma$ was proven in [3] and [4]. The general overview of NP-hardness of computational problems in interval context is given in [8].

\section{How Can We Actually Detect Guaranteed Outliers?}

How can we actually compute these values? First, we will show that if $1+\left(1 / k_{0}\right)^{2}<n$ (which is true, e.g., if $k_{0}>$ 1 and $n \geq 2$ ), then the maximum of $U$ (correspondingly, the minimum of $L$ ) is always attained at some combination of endpoints of the intervals $\mathbf{x}_{i}$; thus, in principle, to determine the values $\bar{U}$ and $\underline{L}$, it is sufficient to try all $2^{n}$ combinations of values $\underline{x}_{i}$ and $\bar{x}_{i}$ :

Theorem 4 If $1+\left(1 / k_{0}\right)^{2}<n$, then the maximum of the function $U$ and the minimum of the function $L$ on the box $\mathbf{x}_{1} \times \ldots \times \mathbf{x}_{n}$ are attained at its vertices, i.e., when for every $i$, either $x_{i}=\underline{x}_{i}$ or $x_{i}=\bar{x}_{i}$.

NP-hard means, crudely speaking, that there are no general ways for solving all particular cases of this problem (i.e., computing $\bar{V}$ ) in reasonable time.

However, we show that there are algorithms for computing $\bar{U}$ and $\underline{L}$ for many reasonable situations. Namely, we propose efficient algorithms that compute $\bar{U}$ and $\underline{L}$ for the case when all the interval midpoints ("measured values") $\widetilde{x}_{i} \stackrel{\text { def }}{=}\left(\underline{x}_{i}+\bar{x}_{i}\right) / 2$ are definitely different from each other, in the sense that the "narrowed" intervals

$$
\left[\widetilde{x}_{i}-\frac{1+\alpha^{2}}{n} \cdot \Delta_{i}, \widetilde{x}_{i}+\frac{1+\alpha^{2}}{n} \cdot \Delta_{i}\right]
$$

- where $\alpha=1 / k_{0}$ and $\Delta_{i} \stackrel{\text { def }}{=}\left(\underline{x}_{i}-\bar{x}_{i}\right) / 2$ is the interval's half-width - do not intersect with each other.

The algorithms $\overline{\mathcal{A}}_{U}$ and $\underline{\mathcal{A}}_{L}$ are as follows: 
- In both algorithms, first, we sort all $2 n$ endpoints of the narrowed intervals $\widetilde{x}_{i}-\frac{1+\alpha^{2}}{n} \cdot \Delta_{i}$ and $\widetilde{x}_{i}+\frac{1+\alpha^{2}}{n}$. $\Delta_{i}$ into a sequence $x_{(1)} \leq x_{(2)} \leq \ldots \leq x_{(2 n)}$. This enables us to divide the real line into $2 n+1$ segments ("small intervals") $\left[x_{(i)}, x_{(i+1)}\right]$, where we denoted $x_{(0)} \stackrel{\text { def }}{=}-\infty$ and $x_{(2 n+1)} \stackrel{\text { def }}{=}+\infty$.

- For each of small intervals $\left[x_{(i)}, x_{(i+1)}\right]$, we do the following: for each $j$ from 1 to $n$, we pick the following value of $x_{j}$ :

- if $x_{(i+1)}<\widetilde{x}_{j}-\frac{1+\alpha^{2}}{n} \cdot \Delta_{j}$, then we pick $x_{j}=$ $\bar{x}_{j}$

- if $x_{(i+1)}>\widetilde{x}_{j}+\frac{1+\alpha^{2}}{n} \cdot \Delta_{j}$, then we pick $x_{j}=$ $\underline{x}_{j}$;

- for all other $j$, we consider both possible values $x_{j}=\bar{x}_{j}$ and $x_{j}=\underline{x}_{j}$.

As a result, we get one or several sequences of $x_{j}$ for each small interval.

- To compute $\bar{U}$, for each of the sequences $x_{j}$, we check whether, for the selected values $x_{1}, \ldots, x_{n}$, the value of $E-\alpha \cdot \sigma$ is indeed within the corresponding small interval, and if it is, compute the value $U=E+k_{0} \cdot \sigma$. Finally, we return the largest of the computed values $U$ as $\bar{U}$.

- To compute $\underline{L}$, for each of the sequences $x_{j}$, we check whether, for the selected values $x_{1}, \ldots, x_{n}$, the value of $E+\alpha \cdot \sigma$ is indeed within the corresponding small interval, and if it is, compute the value $L=E-k_{0} \cdot \sigma$. Finally, we return the smallest of the computed values $L$ as $\underline{L}$.

Theorem 5 Let $1 / n+1 / k_{0}^{2}<1$. The algorithms $\overline{\mathcal{A}}_{U}$ and $\underline{\mathcal{A}}_{L}$ compute $\bar{U}$ and $\underline{L}$ in quadratic time for all the cases in which the "narrowed" intervals do not intersect with each other.

These algorithms also work when, for some fixed $C$, no more than $C$ "narrowed" intervals can have a common point:

Theorem 6 Let $1+\left(1 / k_{0}\right)^{2}<n$. For every positive integer $C$, the algorithms $\overline{\mathcal{A}}_{U}$ and $\underline{\mathcal{A}}_{L}$ compute $\bar{U}$ and $\underline{L}$ in quadratic time for all the cases in which no more than $C$ "narrowed" intervals can have a common point.

The corresponding computation times are quadratic in $n$ but grow exponentially with $C$. So, when $C$ grows, this algorithm requires more and more computation time. It is worth mentioning that the examples on which we prove NPhardness correspond to the case when $n / 2$ out of $n$ narrowed intervals have a common point.

\section{Solution to the Fuzzy-Related Problem: Computing Degree of Outlier-ness}

Formulation of the problem. As we have mentioned in the Introduction, instead of classifying a given value $x$ as an outlier or not an outlier, it is desirable to return a degree to which $x$ is an outlier. As a characteristic of this degree, it is natural to take the largest value $k_{0}$ for which $x$ is outside the corresponding $k_{0}$-sigma interval $\left[E-k_{0} \cdot \sigma, E+k_{0} \cdot \sigma\right]$.

If we know the exact values of the measurement results $x_{1}, \ldots, x_{n}$, then we can compute the exact values of $E$ and $\sigma$ and thus, determine this "degree of outlier-ness" as the ratio $r \stackrel{\text { def }}{=}|x-E| / \sigma$. If we only know the intervals $\mathbf{x}_{i}$ of possible values of $x_{i}$, then different values $x_{i} \in \mathbf{x}_{i}$ may lead to different values of this ratio. In this situation, it is desirable to know the interval of possible values of $r$.

Reduction to a simpler problem. The value of $r$ does not change if, instead of the original variables $x_{i}$ with values from intervals $\mathbf{x}_{i}$, we consider new variables $x_{i}^{\prime} \stackrel{\text { def }}{=} x_{i}-x$ and a new value $x^{\prime}=0$. Indeed, in this case, $E^{\prime}=E-$ $x$ hence $E^{\prime}-x^{\prime}=E-x$, and the standard deviation $\sigma$ does not change if we simply shift all the values $x_{i}$. Thus, without losing generality, we can take assume that $x=0$, and we are interested in the ratio $|E| / \sigma$.

The lower bound of this ratio is attained when the reverse ratio $1 / r=\sigma /|E|$ is the largest, and vice versa. Thus, to find the interval of possible values for $|E| / \sigma$, it is sufficient to find the interval of possible values of $\sigma /|E|$. Computing this interval is, in its turn, equivalent to computing the interval for the square $V / E^{2}$ of the reverse ratio $1 / r$.

Finally, since $V=M-E^{2}$, where $M \stackrel{\text { def }}{=}$ $\frac{x_{1}^{2}+\ldots+x_{n}^{2}}{n}$ is the second moment, we have $V / E^{2}=$ $M / E^{2}-1$, so computing the bounds for $V / E^{2}$ is equivalent to computing the bounds for the ration $R \stackrel{\text { def }}{=} M / E^{2}$. In this section, we will describe how to compute the bounds $\underline{R}$ and $\bar{R}$ for the ratio $R$; based on these bounds, we can compute the desired bounds on $k_{0}$.

Theorem 7 The following algorithm $\underline{\mathcal{A}}_{R}$ always computes $\underline{R}$ in quadratic time.

If all the original intervals have a common point, then the smallest value of $V$ is 0 , and $\underline{R}=1$. If not all $n$ intervals $\mathbf{x}_{i}$ intersect, then, first, we sort all $2 n$ values $\underline{x}_{i}, \bar{x}_{i}$ into a sequence $x_{(1)} \leq x_{(2)} \leq \ldots \leq x_{(2 n)}$; take $x_{(0)}=-\infty$ and $x_{(2 n+1)}=+\infty$. Thus, the real line is divided into $2 n+1$ zones $\left(x_{(0)}, x_{(1)}\right],\left[x_{(1)}, x_{(2)}\right], \ldots,\left[x_{(2 n-1)}, x_{(2 n)}\right]$, $\left[x_{(2 n)}, x_{(2 n+1)}\right)$.

For each of these zones $\left[x_{(k)}, x_{(k+1)}\right], k=0,1, \ldots, 2 n$, we compute the values $e_{k}, m_{k}$, and $n_{k}$ as in Algorithm 
$\underline{\mathcal{A}}_{U}$, then compute the value $\lambda_{k}=m_{k} / e_{k}$. If $\lambda_{k} \in$ $\left[x_{(k)}, x_{(k+1)}\right]$, we compute $R_{k}=M_{k} / E_{k}^{2}$, where

$$
E_{k} \stackrel{\text { def }}{=} \frac{e_{k}+\lambda_{k} \cdot n_{k}}{n} ; M_{k} \stackrel{\text { def }}{=} \frac{m_{k}+\lambda_{k}^{2} \cdot n_{k}}{n} .
$$

The smallest of the corresponding values $R_{k}$ is the desired bound $\underline{R}$.

Computing $\bar{R}$. We are able to compute $\bar{R}$ if the "narrowed" intervals $\left[x_{i}^{-}, x_{i}^{+}\right]$have few intersections, where:

$$
x_{i}^{-} \stackrel{\text { def }}{=} \frac{\widetilde{x}_{i}}{1+\frac{\Delta_{i}}{\underline{E} \cdot n}} ; x_{i}^{+} \stackrel{\text { def }}{=} \frac{\widetilde{x}_{i}}{1-\frac{\Delta_{i}}{\underline{E} \cdot n}},
$$

and $\underline{E} \stackrel{\text { def }}{=} \frac{\underline{x}_{1}+\ldots+\underline{x}_{n}}{n}$.

Theorem 8 For every positive integer $C$, the following algorithm $\overline{\mathcal{A}}_{R}$ computes $\bar{R}$ in quadratic time for all the cases in which no more than $C$ "narrowed" intervals can have a common point.

In this algorithm, we sort $2 n$ values $\underline{x}_{i}$ and $\bar{x}_{i}$ into a sequence $x_{(1)} \leq x_{(2)} \leq \ldots \leq x_{(2 n)}$; take $x_{(0)}=-\infty$ and $x_{(2 n+1)}=+\infty$, and thus divide the real line into $2 n+1$ zones $\left(x_{(0)}, x_{(1)}\right],\left[x_{(1)}, x_{(2)}\right], \ldots,\left[x_{(2 n-1)}, x_{(2 n)}\right]$, $\left[x_{(2 n)}, x_{(2 n+1)}\right)$.

For each of these zones $\left[x_{(k)}, x_{(k+1)}\right], k=0,1, \ldots, 2 n$, and for each variable $x_{i}$, we take:

- $x_{i}=\underline{x}_{i}$ if $x_{i}^{+} \leq x_{(k)}$;

- $x_{i}=\bar{x}_{i}$ if $x_{i}^{-} \geq x_{(k+1)}$;

- both values $x_{i}=\underline{x}_{i}$ and $x_{i}=\bar{x}_{i}$ otherwise.

Since no more than $C$ intervals have a common intersection, for each zone, we thus create have no more than $2^{C}$ different combinations $\left(x_{1}, \ldots, x_{n}\right)$. For each of these combinations, we compute $E$ and $M$ and check whether $M / E$ belongs to this zone. If it belongs, we compute $M / E^{2}$.

The largest of thus computed values $M / E^{2}$ is the desired upper endpoint $\bar{R}$.

\section{Proofs: Main Idea}

Our proof of Theorem 2.1 is based on the fact that when the function $U\left(x_{1}, \ldots, x_{n}\right)$ attains its smallest possible value at some point $\left(x_{1}^{\mathrm{opt}}, \ldots, x_{n}^{\mathrm{opt}}\right)$, then, for every $i$, the corresponding function of one variable

$$
U_{i}\left(x_{i}\right) \stackrel{\text { def }}{=} U\left(x_{1}^{\mathrm{opt}}, \ldots, x_{i-1}^{\mathrm{opt}}, x_{i}, x_{i+1}^{\mathrm{opt}}, \ldots, x_{n}^{\mathrm{opt}}\right)
$$

- the function that is obtained from $U\left(x_{1}, \ldots, x_{n}\right)$ by fixing the values of all the variables except for $x_{i}-$ also attains its minimum at the value $x_{i}=x_{i}^{\mathrm{opt}}$.
A differentiable function of one variable attains its minimum on a closed interval either at one of its endpoints or at an internal point in which its first derivative is equal to 0 .

This first derivative is equal to 0 when $\sigma+k_{0} \cdot\left(x_{i}-E\right)=$ 0 , i.e., when $x_{i}=E-\alpha \cdot \sigma$, where $\alpha=1 / k_{0}$. Thus, for the optimal values $x_{1}, \ldots, x_{n}$ for which $U$ attains its minimum, for every $i$, we have either $x_{i}=\underline{x}_{i}$, or $x_{i}=\bar{x}_{i}$, or $x_{i}=E-\alpha \cdot \sigma$.

We then show that if the open interval $\left(\underline{x}_{i}, \bar{x}_{i}\right)$ contains the value $E-\alpha \cdot \sigma$, then the minimum of the function cannot be attained at points $\bar{x}_{i}$ or $\underline{x}_{i}$ and therefore, has to be attained at the value $x_{i}=E-\alpha \cdot \sigma$.

We also show that:

- when $E-\alpha \cdot \sigma \leq \underline{x}_{i}$, the minimum cannot be attained for $x_{i}=\bar{x}_{i}$ and therefore, it is attained when $x_{i}=\underline{x}_{i}$;

- when $\bar{x}_{i} \leq E-\alpha \cdot \sigma$, the minimum cannot be attained for $x_{i}=\underline{x}_{i}$ and therefore, it is attained when $x_{i}=\bar{x}_{i}$.

Due to what we have proven, once we know how the value $\mu \stackrel{\text { def }}{=} E-\alpha \cdot \sigma$ is located with respect to all the intervals $\left[\underline{x}_{i}, \bar{x}_{i}\right]$, we can find the optimal values of $x_{i}$. Hence, to find the minimum, we need to analyze how the endpoints $\underline{x}_{i}$ and $\bar{x}_{i}$ divide the real line, and consider all the resulting sub-intervals.

\section{Conclusions}

In many application areas, it is important to detect outliers. Traditional engineering approach to outlier detection is that we start with some "normal" values $x_{1}, \ldots, x_{n}$, compute the sample average $E$, the sample standard variation $\sigma$, and then mark a value $x$ as an outlier if $x$ is outside the $k_{0^{-}}$ sigma interval $\left[E-k_{0} \cdot \sigma, E+k_{0} \cdot \sigma\right]$ (for some pre-selected parameter $k_{0}$ ).

In real life, we often have only interval ranges $\mathbf{x}_{i}=$ $\left[\underline{x}_{i}, \bar{x}_{i}\right]$ for the normal values $x_{1}, \ldots, x_{n}$. For different values $x_{i} \in \mathbf{x}_{i}$, we get different values of $L \stackrel{\text { def }}{=} E-k_{0} \cdot \sigma$ and $U \stackrel{\text { def }}{=} E+k_{0} \cdot \sigma-$ and thus, different $k_{0}$-sigma intervals $[L, U]$. We can therefore identify guaranteed outliers as values that are outside all $k_{0}$-sigma intervals, and possible outliers as values that are outside some $k_{0}$-sigma intervals. To detect guaranteed and possible outliers, we must therefore be able to compute the range $\mathbf{L}=[\underline{L}, \bar{L}]$ of possible values of $L$ and the range $\mathbf{U}=[\underline{U}, \bar{U}]$ of possible values of $U$.

In our previous papers $[3,4]$, we have shown how to compute the intervals $\mathbf{E}=[\underline{E}, \bar{E}]$ and $[\underline{\sigma}, \bar{\sigma}]$ of possible values for $E$ and $\sigma$. In principle, we can combine these intervals and conclude, e.g., that $L$ always belongs to the interval $\mathbf{E}-k_{0} \cdot[\underline{\sigma}, \bar{\sigma}]$. However, the resulting interval for $L$ is wider than the actual range - wider because the values $E$ and $\sigma$ are computed based on the same inputs $x_{1}, \ldots, x_{n}$ and are, therefore, not independent from each other. 
If, instead of the actual ranges for $L$ and $U$, we use wider intervals, we may miss some outliers. It is therefore important to compute the exact ranges for $L$ and $U$.

In this paper, we showed that computing these ranges is, in general, NP-hard, and we provided efficient algorithms that compute these ranges under reasonable conditions.

We also provide algorithms that estimate the degree of "outlier-ness" of a given value $x$ - measured as the largest value $k_{0}$ for which $x$ is outside the corresponding $k_{0}$-sigma interval.

\section{Acknowledgments}

This work was supported in part by NASA grants NCC5-209 and grant NCC2-1232, by the Air Force Office of Scientific Research grant F49620-00-1-0365, by NSF grants CDA-9522207, EAR-0112968, EAR-0225670, and 9710940 Mexico/Conacyt, by IEEE/ACM SC2001 and SC2002 Minority Serving Institutions Participation Grants, by Small Business Innovation Research grant 9R44CA81741 to Applied Biomathematics from the National Cancer Institute (NCI), a component of the National Institutes of Health (NIH), and by a grant from Sandia National Laboratories as part of the Department of Energy Accelerated Strategic Computing Initiative (ASCI).

\section{References}

[1] J. Devore and R. Peck, Statistics: the Exploration and Analysis of Data, Duxbury, Pacific Grove, California, 1999.

[2] C. Ferregut, R. A. Osegueda, and A. Nuñez (eds.), Proceedings of the International Workshop on Intelligent NDE Sciences for Aging and Futuristic Aircraft, El Paso, TX, September 30-October 2, 1997

[3] S. Ferson, L. Ginzburg, V. Kreinovich, L. Longpré, and M. Aviles, Computing Variance for Interval Data is NPHard. ACM SIGACT News, 33(2):108-118, 2002.

[4] S. Ferson, L. Ginzburg, V. Kreinovich, and M. Aviles, Exact Bounds on Sample Variance of Interval Data. In: Extended Abstracts of the 2002 SIAM Workshop on Validated Computing, Toronto, Canada, 2002, 67-69

[5] M. Goodchild and S. Gopal, Accuracy of Spatial Databases, Taylor \& Francis, London, 1989.

[6] X. E. Gros, NDT Data Fusion, J. Wiley, London, 1997.

[7] O. Kosheleva, S. Cabrera, R. Osegueda, S. Nazarian, D. L. George, M. J. George, V. Kreinovich, and K. Worden, Case study of non-linear inverse problems: mammography and non-destructive evaluation. In: A.
Mohamad-Djafari (ed.), Bayesian Inference for Inverse Problems, Proceedings of the SPIE/International Society for Optical Engineering, vol. 3459, San Diego, CA, 1998, 128-135.

[8] V. Kreinovich, A. Lakeyev, J. Rohn, and P. Kahl, Computational complexity and feasibility of data processing and interval computations, Kluwer, Dordrecht, 1997.

[9] M. McCain and C. William, Integrating Quality Assurance into the GIS Project Life Cycle. Proceedings of the 1998 ESRI Users Conference http://www.dogcreek.com/html/documents.html

[10] R. Osegueda, V. Kreinovich, L. Potluri, and R. Aló, Non-Destructive Testing of Aerospace Structures: Granularity and Data Mining Approach. Proceedings of FUZZ-IEEE'2002, Honolulu, Hawaii, May 12-17, 2002, Vol. 1, 685-689

[11] R. A. Osegueda, S. R. Seelam, A. C. Holguin, V. Kreinovich, and C.-W. Tao, Statistical and DempsterShafer Techniques in Testing Structural Integrity of Aerospace Structures. International Journal of Uncertainty, Fuzziness, Knowledge-Based Systems (IJUFKS) 9:749-758, 2001.

[12] S. Rabinovich, Measurement Errors: Theory and Practice, American Institute of Physics, New York, 1993.

[13] L. Scott, Identification of GIS Attribute Error Using Exploratory Data Analysis. Professional Geographer 46:378-386, 1994.

[14] S. A. Vavasis, Nonlinear optimization: complexity issues, Oxford University Press, N.Y., 1991.

[15] H. M. Wadsworth, Jr. (ed.), Handbook of statistical methods for engineers and scientists, McGraw-Hill Publishing Co., N.Y., 1990.

[16] Q. Wen, A. Q. Gates, J. Beck, V. Kreinovich, and G. R. Keller, Towards automatic detection of erroneous measurement results in a gravity database. Proceedings of the 2001 IEEE Systems, Man, and Cybernetics Conference, Tucson, Arizona, October 7-10, 2001, 21702175

[17] K. Worden, R. Osegueda, C. Ferregut, S. Nazarian, D. L. George, M. J. George, V. Kreinovich, O. Kosheleva, and S. Cabrera, Interval Methods in Non-Destructive Testing of Material Structures. Reliable Computing 7:341-352, 2001. 